\title{
Periprosthetic fungal infections: Be alert Clinical cases and review of the literature
}

\author{
Correspondence \\ Dr L Botha \\ Department of Orthopaedics \\ 1 Military Hospital \\ Thaba Tshwane \\ Pretoria \\ South Africa \\ Tel: +27 123140044
}

Dr Leonore Botha* ${ }^{*} B C h B(P r e t)$, MMed Orth(Pret), FC Ortho(SA)

Prof Theo LB le Roux* MBChB(Pret), MMed Orth(Pret), FC Ortho(SA)

Dr Heather McLoughlin** MBibl/MA (Psych)RAU/D Litt et Phil (Psych)UJ

${ }^{*}$ Department of Orthopaedics, 1 Military Hospital, University of Pretoria, Faculty of Health Sciences, School of Medicine, South Africa

${ }^{* *}$ Clinical psychologist, Information Consultant, Pretoria East Hospital

Email: leonoreduplessis@gmail.com

\begin{abstract}
The increasing rate of arthroplasty, revisions and resistance to antibiotics has increased the risk for fungal infections. Different treatment modalities exist: suppressive therapy, debridement with retained prosthesis, Girdlestone procedures and 2- or even 3-stage revision arthroplasty. Fungal infections after joint replacements are rare but devastating.

Purpose of the study: The aim was to see if there was any trend that could help with the diagnosis and management of patients with fungal infections. The literature was reviewed in order to assist with diagnosis and treatment.

Materials and methods: A retrospective study was performed and all the cases seen and treated by a tumour and sepsis orthopaedic specialist from 1999 to 2015 were evaluated. Inclusion criteria: Patients had to be diagnosed with a fungal infection in any specimen which was sent for histology or culture. Exclusions: none.

Results: Four patients were identified. All of them were males. Mean age 58 (35-71) years. The primary surgical indications were: vertebral osteomyelitis; post primary knee replacement; pig bite with lower limb sepsis and osteoarthritis knee, and a septic total hip replacement. Three cases cultured Candida parapsilosis and one Candida albicans of which three were tissue cultures and one a blood culture.

Currently 75\% have failed treatment - one passed away, one developed systemic sepsis, and one had an above-knee amputation and is still struggling with subsequent bacterial infections in the amputation stump.

Conclusion: As long as there are higher incidences of fungal infections with devastating complications more evidence is needed. Numerous small case studies have been published, with the purpose of looking for the correct treatment: monotherapy, combination therapy, newer antifungals, higher dosages, implant retention or removal. It appears that the correct answer is unclear as yet.

It is important to always have a high index of suspicion and good pre-operative planning together with a team approach: infectious diseases specialist, microbiologist and histologist. This approach will optimise the probability of making a diagnosis and to appropriately manage the fungus cultured.
\end{abstract}

Key words: periprosthetic infection, fungal infection, antifungals, Candidiasis, sepsis

http:/ / dx.doi.org/10.17159/2309-8309/2016/v15n2a1

\section{Introduction}

The increasing rate of arthroplasty, revisions and resistance to antibiotics has increased the risk for fungal infections. Fungal infections after joint replacements are rare but devastating. Different treatment modalities exist: suppressive therapy, debridement with retained prosthesis, Girdlestone procedures and 2- or even 3-stage revision arthroplasty. The aim of the review is to guide the surgeon with regard to a protocol to address fungal infections. 


\section{Materials and method}

A retrospective study was performed and all the cases seen and treated by a Tumour and Sepsis orthopaedic specialist from 1999 to 2015 were evaluated.

Inclusion criteria: The patient had to be diagnosed with a fungal infection in any specimen that was sent for histology or culture. There were no exclusions.

The aim was to see if there was any trend that could help make the diagnosis and management of these patients more successful. In this study there were four patients who were diagnosed with a fungal infection.

\section{Outcomes}

The four patients who were identified were all males, with a mean age of $58(35-71)$ years. The primary surgical indications were: vertebral osteomyelitis; post primary knee replacement; pig bite with lower limb sepsis and osteoarthritis knee, and a septic total hip replacement. None were treated for the same reason and they were not initially treated by the tumour and sepsis surgeon. Three cultured Candida parapsilosis and one Candida albicans, of which three were tissue cultures and one a blood culture.

One patient had a primary total knee replacement for osteoarthritis and cultured Candida parapsilosis on blood culture sensitive to amphotericin $B$ and caspofungin and resistant to fluconazole. He did not go back to theatre and was treated with IV caspofungin for 2 weeks. His current follow-up is only one month.

The second patient had vertebral osteomyelitis and cultured a Candida parapsilosis on tissue sensitive to caspofungin and amphotericin B, resistant to fluconazole. He had numerous debridements and antifungal treatment. He was on IV caspofungin for 6 months. To date he has had 14 admissions, systemic uncontrolled sepsis and a high morbidity.

The third patient presented with a pig bite and developed severe sepsis at the site of the bite. Candida albicans was cultured. It responded to Diflucan. He had a total knee replacement 4 months later which was complicated by recurrent fungal infection. He ended up with an amputation 3 years later and is still struggling with recurrent bacterial infections in the amputation stump.

The fourth patient had a septic total hip replacement. The hip replacement was performed in 2010 . He presented with subsequent sepsis. He had numerous debridements including removal of the prosthesis. Candida parapsilosis was cultured from a tissue specimen. It was sensitive to all the antifungal agents and was treated with amphotericin B and fluconazole for 6 months. Subsequently the patient passed away.

After evaluating these four patients a definite trend could not be detected. Up to now treatment has failed in three. One of them passed away. One developed systemic sepsis and one had an above-knee amputation. Despite the amputation he is still struggling with subsequent bacterial infections. The only one with no morbidity had his last intervention one month ago. It is known that fungal infections usually present late. The poor outcome of fungal musculoskeletal infection was the motivation for this review.

The cases were summarised (Table I) according to the primary surgical indication, co-morbidities, organism cultures, surgical history and intervention.

\begin{tabular}{|c|c|c|c|c|}
\hline & Patient 1 & Patient 2 & Patient 3 & Patient 4 \\
\hline Age/Sex & $71 / \mathrm{M}$ & $58 / \mathrm{M}$ & $35 / \mathrm{M}$ & $69 / \mathrm{M}$ \\
\hline $\begin{array}{l}\text { Primary surgical } \\
\text { indication }\end{array}$ & Osteoarthritis & $\begin{array}{c}\text { Vertebral } \\
\text { osteomyelitis }\end{array}$ & $\begin{array}{l}\text { Pig bite sepsis knee and } \\
\text { leg } 8 \text { months before TKR }\end{array}$ & Sepsis THR \\
\hline Co-morbidities & $\begin{array}{l}\text { Hypertension, } \\
\text { hypothyroidism }\end{array}$ & Bladder cancer & None & Testosterone injections \\
\hline Organism & Candida parapsilosis & Candida parapsilosis & Candida albicans & Candida parapsilosis \\
\hline Prior surgery & THR 2001 unrelated & $\begin{array}{l}\text { Bladder CA } 2 \\
\text { years before }\end{array}$ & Debridement elsewhere & $\begin{array}{l}\text { Debrided for perianal sepsis } 1 \\
\text { year before THR and debridement } \\
2 \text { years post THR elsewhere }\end{array}$ \\
\hline Rx surgical & $\begin{array}{l}\text { TKR unaware } \\
\text { of infection }\end{array}$ & $\begin{array}{l}\text { Debridement - } \\
\text { numerous }\end{array}$ & Debridement and specimens & $\begin{array}{l}\text { Removal of prosthesis and cement } \\
\text { spacer } 3 \text { years post replacement }\end{array}$ \\
\hline Bone cement & None & None & Cement spacer with gentamycin & Cement spacer with gentamycin \\
\hline Time between stages & None & None & 5 months & 1 year and 6 months \\
\hline $\begin{array}{l}\text { Systemic } \\
\text { antimicrobial } \mathrm{Rx}\end{array}$ & Caspofungin for $2 / 52$ & $\begin{array}{l}\text { Caspofungin } \mathrm{f} \\
\text { or } 6 / 12\end{array}$ & $\begin{array}{l}\text { Diflucan IVI for } 1 / 52 \\
\text { then oral for } 6 / 52\end{array}$ & $\begin{array}{l}\text { Amphotericin B followed by } \\
\text { fluconazole }\end{array}$ \\
\hline Follow-up & 1 month & $\begin{array}{l}1 \text { year currently } \\
\text { still septic }\end{array}$ & $\begin{array}{l}\text { Sepsis recurred after TKR and had a } \\
\text { amputation } 3 \text { years later, still struggling } \\
\text { with recurrent bacterial sepsis }\end{array}$ & $\begin{array}{l}\text { Recurrent sepsis after } \\
5 \text { months, passed away }\end{array}$ \\
\hline
\end{tabular}




\section{Discussion}

Infection occurs in 1-2\% of primary hip and knee replacements, and in revision cases the occurrence is $5-40 \%{ }^{1}$ Fungal infections are rare and occur in approximately $1 \%$ of all periprosthetic joint infections. ${ }^{2}$

The trend towards fungal infections in the immunosuppressed host appears to be higher than in the healthy host. These include patients with: malignant disease, drug therapies (antineoplastic, corticosteroids, immunosuppressive drugs), overuse of antibiotics, indwelling catheters, diabetes, TB, multiple revision surgery, reconstructions, and in IV drug users. ${ }^{3} \mathrm{~A}$ high index of suspicion must, however, still be maintained in immunocompetent hosts. Fungal infections in immunocompetent hosts have been reported so do not just regard the specimen as being contaminated. ${ }^{4}$

The organisms mostly involved are: Candida (albicans, parapsilosis, glabrata, tropicalis), Aspergillus, Coccidioides and Blastomycetes. ${ }^{3,5}$ Candida albicans is the most common organism. ${ }^{6}$

Fungal infections are prone to biofilm formation which makes treatment difficult as the drugs available do not all have good biofilm penetration. Candida albicans especially produces a more complex biofilm which increases the antifungal resistance. ${ }^{7}$

The more widespread use of antifungals increases the resistance of organisms, thus only use it for specific indications. ${ }^{8}$

The mortality rate can be as high as $25 \%$ in periprosthetic fungal infections. ${ }^{2}$

\section{Clinical picture}

The patients with fungal infections do not typically present with the same symptoms as those with bacterial prosthetic joint infections. The symptoms are usually longstanding and can be: pain, erythema, swelling and decreased range of movement. Fever and a fistula are rare. ${ }^{3,9-11}$ The mean timespan between surgery and clinical signs is 21 months. $^{9}$

\section{Diagnosis}

Standard special investigations for bacterial prosthetic joint infection (leucocytes, ESR, CRP) are of little value. ${ }^{9,10}$ PCR might be an alternative, but is not proven yet. ${ }^{10}$

We are unable to differentiate bacterial from fungal infections on systemic inflammatory markers and aspiration cell count. ${ }^{12}$ Prosthetic loosening is common. ${ }^{3,10,11,13}$

A diagnostic error is often made by saying it is only a contaminant, whereas it might be the reason for the patient's symptoms. ${ }^{9,10,11}$ Kuiper et al..$^{10}$ had $51 \%$ of their original cultures reported as possible contaminants but in the end $21 \%$ of them were falsely reported as contaminants.

Gram stain is rarely positive. ${ }^{3,9,11,14}$ Currently we rely on the results of cultures from joint aspirations or tissue. It can be intra-operative tissue specimens or aspirations., ${ }^{3,10,11}$
Cultures should not be accepted as being negative until 4 weeks after incubation. ${ }^{3}$ Always remember to check other cultures (blood and urinary cultures). If negative cultures are reported but the clinician still thinks it might be fungal, repeat the aspiration. Thirty per cent of reported cases had to have repeat aspirations before they were proven positive. ${ }^{15}$

Negative histological examinations have been reported where re-examination with another stain showed a positive fungal infection. ${ }^{13}$

To date there is no guideline on the number of samples needed or on the amount that needs to be positive. However, it is so difficult to get a positive culture that any indication should rather be considered as being positive than a contaminant. ${ }^{9,10}$ One study suggested that at least two positive cultures are needed. ${ }^{2}$ Fungal infection is however so devastating that the tumour and sepsis specialist involved in this study acts on one positive culture.

Fungal organisms can be negative in synovial fluid or purulent secretions. It is difficult to detect if the samples are not cultured correctly and special staining techniques are not used. $^{3}$

If a patient has skin lesions, tissue of these lesions can be sent for culture and histopathology. ${ }^{16,17}$

Sonification and vortexing of implants is an option. Sonification is more sensitive than vortexing (60\% vs $40 \%$ ), with the same specificity: $99 \% .^{18}$ Remember the risk of contamination of the specimen.

Dutronc et al. ${ }^{9}$ reported in their literature review that immunological tests (immunoelectrophoresis, electrosyneresis and ELISA tests), detection of circulating mannantype antigens and detection of Candida metabolites are rarely used for prosthetic fungal infections. Alternative testing methods are: immunohistochemistry, in situ hybridisation, matrix-assisted laser desorption/ionisation time-offlight (MALDI-TOF), mass spectrometry, and analysis with polymerase chain reaction (PCR)-based procedures. ${ }^{15}$ These are however not always available.

The question raised was whether fungal cultures should be requested in all our prosthetic joint infections and revisions. Two studies stated that it was too expensive and the positive cultures rare. ${ }^{19,20}$ The tumour and sepsis specialist involved in this study is of the opinion that specimens should be sent when there is suspicion of any infection as fungal infections are devastating.

\section{Treatment}

The host needs to be assessed in order to determine if he is fit for surgery. Poor hosts or those refusing surgery should be informed that isolated suppressive therapy is an option but the prognosis is poor. There is a high risk of failure. ${ }^{21}$

It is important to work closely with the infectious disease specialist, physician and microbiologist from the start. The infectious diseases specialist will know the hospital resistance patterns and can monitor the MIC (minimal inhibitory concentration). If there is a difference between clinical response and lab testing the histologist can be 
asked for a histological control when treatment is started and new specimens are to be sent in. Lerch et al. ${ }^{13}$ found marked swelling of the Candida pseudomyelia and oval blastospores after 3-4 days of therapy. The quantity and stainability of cells reduces after 1-2 weeks.

There are different anti-fungal agents available and there are different regimens of treatment for Candidiasis, for example amphotericin B alone, fluconazole alone or these two drugs combined. Caspofungin combined with amphotericin B and flucytosine and voriconazole is also used..$^{22}$

Aspergillosis treatment is mainly voriconazole for Aspergillosis terreus and amphotericin B or voriconazole for non-terreus aspergillosis. ${ }^{22}$ Voriconazole resistance by Aspergillosis is rising. Furustrand et al. ${ }^{23}$ described a rapid calometric assay for detection of voriconazole-resistant Aspergillus fumigatus within 8 hours; this is an option in the sick patient not responding to treatment.

Aspergillus fumigatus is rare. Surgical treatment combined with antifungal therapy is recommended..$^{24}$ Other unusual fungi are Rhodotorula minuta, Aureibasidium, Histoplasma capsulatum and Sporothrix schenckii. ${ }^{3,24}$ It is important to request fungal cultures in addition to aerobic and anaerobic bacterial cultures. ${ }^{24}$

Fluconazole has good joint penetration whether given orally or via intravenous infusion. ${ }^{25}$ Some studies showed good effect as suppressive therapy alone, though most gave it lifelong. ${ }^{11,26-29}$ Numerous studies also proved high failure rates with only suppressive therapy. ${ }^{14,21}$ It has no serious side effects, rapid oral absorption, high bioavailability, and can be given once daily. They also reported a high concentration of fluconazole in joint fluid. ${ }^{25}$ However we found $50 \%$ of the yeasts in the study to be resistant to fluconazole.

Fluconazole and voriconazole were shown to be unable to prevent biofilm formation or eradicate an organism from an existing biofilm in Cryptococcus neoformans; this was an in vitro study. The biofilms were sensitive to amphotericin $B$ and caspofungin but more resistant especially if the biofilm had melanin. They came to the conclusion that biofilm formation might reduce the effect of antifungal drugs in cryptococcal infection..$^{30}$

Amphotericin B is a very toxic drug with serious side effects: immediate infusion- related reactions of hyperpyrexia, severe malaise and hypotension, renal failure, anaemia, hypokalaemia, leucopaenia and thrombocytopaenia were reported. ${ }^{31}$

Different forms of Amphotericin B are available. Amphotericin B deoxycholate (AmBd) is the original drug. Side effects however lead to a search for a safer drug. Amphotericin B lipid formulations (ABLC) (amphotericin B lipid complex, amphotericin B colloidal dispersion and liposomal amphotericin $\mathrm{B}$ ) have less renal toxicity.

Kleinberg et al..$^{32}$ found that AmBd, ABLC, fluconazole, voriconazole and caspofungin are equally effective in Candidiasis. In Aspergillosis, however, voriconazole and ABLC are better. ${ }^{32}$

Parvizi et al. published their results. Of 31 patients with fungal infections, 24 were treated with removal of the prosthesis; the remaining seven had an irrigation and debridement of which five failed and ended up with removal of the prosthesis, and the remaining two had severe bone loss and were only treated with suppressive antibiotics. $^{3}$

Of the 29 patients who had further surgery three had a knee arthrodesis, three had above-knee amputation, three had permanent resection arthroplasty, one had a hip disarticulation and 19 had delayed implantation. Of these 19, ten had recurrent infection ending up with amputations, suppressive treatment or death. Thus only nine had a good outcome. This shows how devastating fungal infections can be and that debridement alone is not adequate. ${ }^{3}$

\section{What antifungal should be given? Which dosage?}

Clinical practice guidelines for Candidiasis were published by the Infectious Diseases Society of America in $2009 .{ }^{33}$ The European Society for Clinical Microbiology and Infectious disease published their guidelines in 2012. ${ }^{34}$

Systemic antifungal agents can be divided into four groups: the polyenes (amphotericin B substrates); triazoles (fluconazole, itraconazole, voriconazole and posaconazole); echinocandins (caspofungin, anidulafungin and micafungin) and flucytosine. ${ }^{33}$

The American Society recommends removal of the prosthesis when bone infection is associated with an implant and treatment for at least 6 weeks with fluconazole (400 mg (6 mg/ kg) daily, or lipid formulation amphotericin B 3-5 mg/ $\mathrm{kg}$ daily for at least 2 weeks followed by fluconazole $400 \mathrm{mg}$ daily for 6-12 months or AmB-d/echinocandin $0.5-1 \mathrm{mg} / \mathrm{kg}$ daily for at least 2 weeks followed by fluconazole $400 \mathrm{mg}$ daily for 6-12 weeks. Chronic suppression with fluconazole is recommended if the device cannot be removed. ${ }^{33}$

The European Society recommends treatment with fluconazole if the organism is susceptible. First start with a lipid-based amphotericin B for 2 weeks followed by fluconazole for 4 weeks. Treatment should be for a minimum of 4 weeks. The prosthesis should be removed, but if this is not possible, lifelong fluconazole should be given as suppressive therapy. ${ }^{34}$

The American and European guidelines are quite similar and it is recommended reading for both the surgeon and infection diseases specialist.

An in vitro study was done by Maiolo et al..$^{35}$ They found that antifungal activities against Candidiasis can be measured with microcalorimetry. Fluconazole, caspofungin, anidulafungin and amphotericin B were tested. Higher concentrations of fluconazole were needed to inhibit Candida biofilm formation than for the planktonic Candida. Caspofungin and amphotericin B kept their activity against biofilms. Anidulafungin had the best antibiofilm activity.

Lerch et al. ${ }^{13}$ had a case that did not respond to fluconazole $400 \mathrm{mg}(6 \mathrm{mg} / \mathrm{kg})$ daily; the patient however responded to $800 \mathrm{mg}$ daily $(12 \mathrm{mg} / \mathrm{kg})$ on histology and clinical. 
Numerous studies proved a good outcome with removal of the implant, antifungals and delayed reimplantation. ${ }^{28,36,37}$

The duration of treatment is uncertain. Make sure negative cultures are confirmed before reinsertion. Prolonged treatment and negative cultures are important. Ueng et al. ${ }^{2}$ recommended at least 3 months of oral fluconazole after the first stage resection arthroplasty, followed by 6 weeks treatment after reimplantation of the prosthesis.

A minimum of one year of treatment was suggested. ${ }^{25,27}$ The IDSA guidelines suggest $6-12$ months. ${ }^{33}$

\section{Combined or monotherapy $?^{38}$}

Echinocandins are an option as they have a wide spectrum of activity, good biofilm penetration, and fewer side effects. ${ }^{1,39}$

A combination of fluconazole with echinocandins and micafungin was recommended as a good option in one case. $^{14}$

Caspofungin and flucytosine is another option for combination therapy. ${ }^{39}$

\section{What should the period be before reinsertion?}

A negative clinical picture and negative cultures after long follow-up should be achieved before reinsertion is considered.

How long is long enough? Phelan et al. ${ }^{37}$ evaluated a total of ten patients and suggested delayed implantation (median of 8.6 [hip] and 2.3 [knee] months, respectively) but they must receive appropriate antifungal treatment.

They found a $20 \%$ recurrence rate of fungal infections in patients with delayed implantation..$^{37}$

Numerous studies suggest delayed (months to years) recurrence of fungal infections in total joint arthroplasty. ${ }^{13,40}$

\section{Cement spacer with or without antifungals?}

Antibiotic-impregnated cement has been shown to work for microbial infections. ${ }^{41,42}$

A few studies were published examining the effect of antifungals on the cement strength, and the release of the drug.

It has been suggested that amphotericin B should be added to cement for its local effect rather than for systemic treatment. This is due to the prevalence of systemic side effects. ${ }^{6}$ Amphotericin is also heat stable which is important for the cement reaction.

Kweon et al. ${ }^{43}$ did a study to compare adding $200 \mathrm{mg}$ amphotericin B to cement (Simplex P) versus adding a high dose poragen (10 g cefazolin) to increase release. They found that the release was increased in the batch with poragen, but it decreased compressive strength significantly. There are also other studies that showed release of amphotericin B mixed with cement. ${ }^{31,44,45}$

Studies differ with regards to amphotericin B release from cement. ${ }^{6,31}$
Goss et al. ${ }^{6}$ found that adding more than $100 \mathrm{mg}$ increases in the compressive strength by about $20 \%$. They did not find any amphotericin B being released from the PMMA due to the chemical binding, thus increasing the strength. They hypothysed that they used Simplex and that the chlorophyll might have had an effect on the binding; however, they could not prove it.

This is not what Silverberg and Marra et al. found, however. ${ }^{31}$ In their experience amphotericin B was released from cement in an in vivo study. ${ }^{31}$ When mixed with Palacos it could be detected in serum and wound fluid for up to 50 hours after implantation. Levels of $3.2 \mathrm{mg} / \mathrm{L}$ were measured. Silverberg et al. ${ }^{46}$ did an in vitro study and incorporated fluconazole, amphotericin B and 5-flucytosine in bone cement. They found that fluconazole and amphotericin remained active whereas 5-flucytosine did not. Inhibitory activity improved with higher drug levels and more drug was eluted from Palacos than Simplex P cement.

Wu et al. ${ }^{47}$ had a good outcome with one patient who could not receive oral amphotericin B. They gave fluconazole IV for 6 weeks followed by oral for 9 weeks; and mixed amphotericin B into the cement spacer (1.2 g of amphotericin B in 40 $\mathrm{g}$ of PMMA). At the revision surgery (6 months later) they still added vancomycin and amphotericin B to the cement with no relapse after 12 months.

Deelstra et $a l .{ }^{4}$ impregnated their cement with voriconazole, amphotericin B and vancomycin. They looked at the serum and drain fluid levels of voriconazole and amphotericin B at 24, 48 and 72 hours. They found detectable levels of these drugs for up to 72 hours. The choice of antifungal agent was based on expert opinion. When the amount of antifungal added to the cement was increased the cement took longer to harden and it became brittle. When used in a spacer they recommended that the patient should be kept non-weight bearing because of concern about the strength of the cement.

Other studies done looked at the efficacy of hydroxyapatite composite impregnated with amphotericin B. They found a superior antifungal efficacy over those in PMMA in an in vitro study. ${ }^{44}$

No study however commented on the safe dose and local release of amphotericin B.

\section{Recommendation}

Always approach the patient the patient as you would any periprosthetic infection. Ask the following questions:

- Is the patient septic?

- What is the organism? Subtype?

- What is the sensitivity?

- Do the treatments' positive effects outweigh the possible treatment complications?

- What it the resistance pattern in the hospitals I work in?

\section{Conclusion}

As long as there is any incidence of fungal infections with devastating complications, more evidence is needed. 


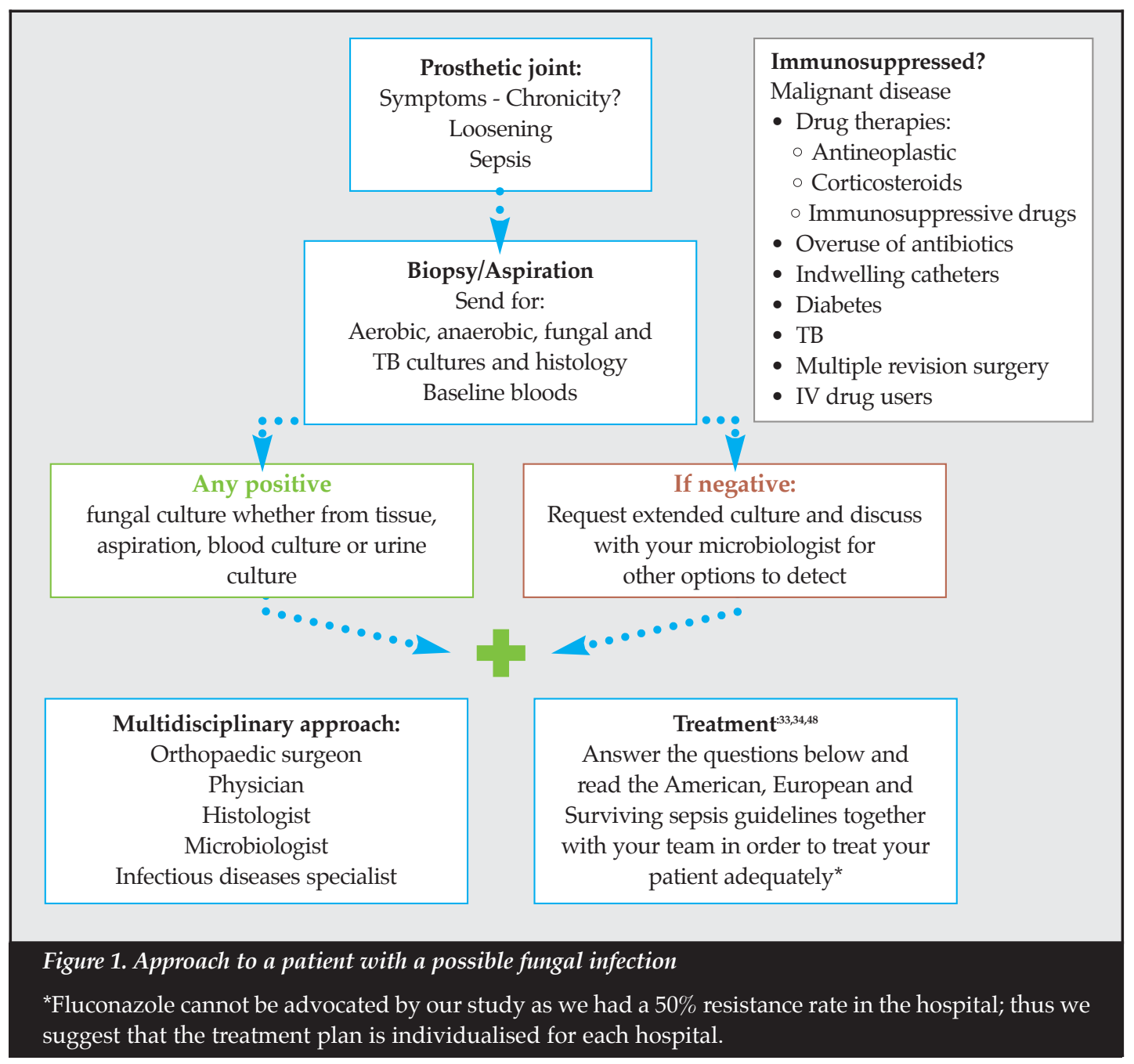

Antimicrobial treatment is often used for prosthetic joint infection leading to increased occurrences of fungal infections. Microcalorimetry is an option to monitor treatment response, resistance and options for combination therapy. An in vivo study would be a good future study in order to monitor treatment efficacy. Numerous small case studies have been published and everybody is looking for the correct treatment: monotherapy, combination therapy, newer antifungals, higher dosages, implant retention or removal. It all points to the fact that the correct answers have not been found as yet.

Making the diagnosis poses the greatest dilemma. The treatment is another issue altogether. It is crucial to have a high index of suspicion and good pre-operative planning together with the team consisting of the orthopaedic surgeon, the infectious diseases specialist, microbiologist and histologist. Figure 1 serves as a guideline as to how to approach a patient with a possible fungal infection. The team approach will optimise the probability of making a diagnosis and lead to appropriately managing the fungus cultured.

\section{Compliance with Ethics Guidelines}

The content of the article is the sole work of the authors. L Botha, TLB le Roux and H McLoughlin declare that no benefits of any form have been or are to be received from a commercial party related directly or indirectly to the subject of the article. The research has not been approved by an ethical committee.

\section{References (recommended reading underlined)}

1. Trampuz A, Widmer AF. Infections associated with orthopedic implants. Curr.Opin.Infect.Dis. 2006 Aug;19(4):349-56.

2. Ueng SW, Lee CY, Hu CC, Hsieh PH, Chang Y. What is the success of treatment of hip and knee candidal periprosthetic joint infection?. Clin.Orthop. 2013 Sep;471(9):3002-3009.

3. Azzam K, Parvizi J, Jungkind D, Hanssen A, Fehring T, Springer $\mathrm{B}$, et al. Microbiological, clinical, and surgical features of fungal prosthetic joint infections: a multi-institutional experience. J.Bone Joint Surg.Am. 2009 Nov;91(Suppl 6):142-49. 
4. Deelstra JJ, Neut D, Jutte PC. Successful treatment of Candida albicans-infected total hip prosthesis with staged procedure using an antifungal-loaded cement spacer. J.Arthroplasty 2013 Feb;28(2):374.e5-374.e8.

5. Inman RD, Gallegos KV, Brause BD, Redecha PB, Christian CL. Clinical and microbial features of prosthetic joint infection. Am.J.Med. 1984 July;77(1):47-53

6. Goss B, Lutton C, Weinrauch P, Jabur M, Gillett G, Crawford $R$. Elution and mechanical properties of antifungal bone cement. J.Arthroplasty 2007 Sep;22(6):902-908.

7. Chandra J, Kuhn DM, Mukherjee PK, Hoyer LL, McCormick T, Ghannoum MA. Biofilm formation by the fungal pathogen Candida albicans: development, architecture, and drug resistance. J.Bacteriol. 2001 Sep;183(18):5385-94.

8. Maschmeyer G. The changing epidemiology of invasive fungal infections: new threats. Int.J.Antimicrob.Agents 2006 Jun;27(Suppl 1):3-6.

9. Dutronc H, Dauchy FA, Cazanave C, Rougie C, LafarieCastet S, Couprie B, et al. Candida prosthetic infections: case series and literature review. Scand.J.Infect.Dis. 2010 Dec;42(11-12):890-95.

10. Kuiper JW, van den Bekerom MP, van der Stappen J, Nolte PA, Colen S. 2-stage revision recommended for treatment of fungal hip and knee prosthetic joint infections. Acta Orthop. 2013 Dec;84(6):517-23.

11. Kelesidis T, Tsiodras S. Candida albicans prosthetic hip infection in elderly patients: is fluconazole monotherapy an option? Scand.J.Infect.Dis. 2010;42(1):12-21.

12. Bracken $C D$, Berbari EF, Hanssen $A D$, Mabry TM, Osmon DR, Sierra RJ. Systemic inflammatory markers and aspiration cell count may not differentiate bacterial from fungal prosthetic infections. Clin.Orthop. 2014 Nov;472(11):3291-94.

13. Lerch K, Kalteis T, Schubert T, Lehn N, Grifka J. Prosthetic joint infections with osteomyelitis due to Candida albicans. Mycoses 2003 Dec;46(11-12):462-66.

14. Bland CM, Thomas S. Micafungin plus fluconazole in an infected knee with retained hardware due to Candida albicans. Ann.Pharmacother. 2009 Mar;43(3):528-31.

15. Gebauer M, Frommelt L, Achan P, Board TN, Conway J, Griffin W, et al. Management of fungal or atypical periprosthetic joint infections. J.Orthop.Res. 2014 Jan;32(Suppl 1):S147-51.

16. Anagnostakos K, Kelm J, Schmitt E, Jung J. Fungal periprosthetic hip and knee joint infections clinical experience with a 2-stage treatment protocol. J.Arthroplasty 2012 Feb;27(2):29398.

17. Gonzalez Santiago TM, Pritt B, Gibson LE, Comfere NI. Diagnosis of deep cutaneous fungal infections: correlation between skin tissue culture and histopathology. J.Am.Acad.Dermatol. 2014 Aug;71(2):293-301.

18. Portillo ME, Salvado M, Trampuz A, Plasencia V, Rodriguez-Villasante $\mathrm{M}$, Sorli $\mathrm{L}$, et al. Sonication versus vortexing of implants for diagnosis of prosthetic joint infection. J.Clin.Microbiol. 2013 Feb;51(2):591-94.

19. Wadey VM, Huddleston JI, Goodman SB, Schurman DJ, Maloney WJ, Baron EJ. Use and cost-effectiveness of intraoperative acid-fast bacilli and fungal cultures in assessing infection of joint arthroplasties. J.Arthroplasty 2010 Dec;25(8):1231-34.
20. Tokarski AT, O'Neil J, Deirmengian CA, Ferguson J, Deirmengian GK. The routine use of atypical cultures in presumed aseptic revisions is unnecessary. Clin.Orthop. 2013 Oct; 471(10):3171-77.

21. Johannsson B, Callaghan JJ. Prosthetic hip infection due to Cryptococcus neoformans: case report. Diagn. Microbiol. Infect.Dis. 2009 May;64(1):76-79.

22. Chandrasekar P. Selection criteria for antifungals: the right patients and the right reasons. International Journal of Antimicrobial Agents. 2006 Jun;27 Suppl 1:17-20.

23. Furustrand Tafin U, Meis JF, Trampuz A. Microcalorimetry assay for rapid detection of voriconazole resistance in Aspergillus fumigatus. Antimicrobial Agents \& Chemotherapy 2013 Nov;57(11):5704-06.

24. Marculescu CE, Berbari EF, Cockerill FR 3rd, Osmon DR. Fungi, mycobacteria, zoonotic and other organisms in prosthetic joint infection. Clin.Orthop. 2006 Oct;451:64-72.

25. Cushing RD, Fulgenzi WR. Synovial fluid levels of fluconazole in a patient with Candida parapsilosis prosthetic joint infection who had an excellent clinical response. J.Arthroplasty 1997 Dec;12(8):950.

26. Penk A, Pittrow L. Role of fluconazole in the long-term suppressive therapy of fungal infections in patients with artificial implants. Mycoses 1999;42(Suppl 2):91-96.

27. Fukasawa N, Shirakura K. Candida arthritis after total knee arthroplasty-a case of successful treatment without prosthesis removal. Acta Orthop.Scand. 1997 Jun;68(3):306-307.

28. Merrer J, Dupont B, Nieszkowska A, De Jonghe B, Outin H. Candida albicans prosthetic arthritis treated with fluconazole alone. J.Infect. 2001 Apr;42(3):208-209.

29. Simonian PT, Brause BD, Wickiewicz TL. Candida infection after total knee arthroplasty. Management without resection or amphotericin B. J.Arthroplasty 1997 Oct;12(7):825-29.

30. Martinez LR, Casadevall A. Susceptibility of Cryptococcus neoformans biofilms to antifungal agents in vitro. Antimicrobial Agents \& Chemotherapy 2006 Mar;50(3):1021-33.

31. Marra F, Robbins GM, Masri BA, Duncan C, Wasan KM, Kwong EH, et al. Amphotericin B-loaded bone cement to treat osteomyelitis caused by Candida albicans. Can.J.Surg. 2001 Oct;44(5):383-86.

32. Kleinberg $\mathrm{M}$. What is the current and future status of conventional amphotericin B? Int.J.Antimicrob.Agents 2006 Jun;27(Suppl 1):12-16.

33. Pappas PG, Kauffman CA, Andes D, Benjamin DK Ir, Calandra TF, Edwards JE Jr, et al. Clinical practice guidelines for the management of candidiasis: 2009 update by the Infectious Diseases Society of America. Clin Infect Dis. 2009;48(5):503-35.

34. Cornely OA, Bassetti M, Calandra T, Garbino I, Kullberg BI, Lortholary O, et al. ESCMID* guideline for the diagnosis and management of Candida diseases 2012: non-neutropenic adult patients. Clin.Microbiol.Infect. 2012 Dec;18(Suppl 7):1937.

35. Maiolo EM, Furustrand Tafin U, Borens O, Trampuz A. Activities of fluconazole, caspofungin, anidulafungin, and amphotericin B on planktonic and biofilm Candida species determined by microcalorimetry. Antimicrobial Agents $\mathcal{E}$ Chemotherapy 2014 May;58(5):2709-17.

36. Lazzarini L, Manfrin V, De Lalla F. Candidal prosthetic hip infection in a patient with previous candidal septic arthritis. J.Arthroplasty 2004 Feb;19(2):248-52. 
37. Phelan DM, Osmon DR, Keating MR, Hanssen AD. Delayed reimplantation arthroplasty for candidal prosthetic joint infection: a report of 4 cases and review of the literature. Clin.Infect.Dis. 2002 Apr 1;34(7):930-38.

38. Fohrer C, Fornecker L, Nivoix Y, Cornila C, Marinescu C, Herbrecht R. Antifungal combination treatment: a future perspective. Int.J.Antimicrob.Agents 2006 Jun;27(Suppl 1):2530.

39. Dumaine V, Eyrolle L, Baixench MT, Paugam A, Larousserie F, Padoin C, et al. Successful treatment of prosthetic knee Candida glabrata infection with caspofungin combined with flucytosine. Int.J.Antimicrob. Agents 2008 Apr;31(4):398-99.

40. Cardinal E, Braunstein EM, Capello WN, Heck DA. Candida albicans infection of prosthetic joints. Orthopedics 1996 Mar;19(3):247-51.

41. Ridgeway S, Wilson J, Charlet A, Kafatos G, Pearson A, Coello R. Infection of the surgical site after arthroplasty of the hip. J. Bone Joint Surg Br. 2005 Jun;87(6):844-50.

42. Espehaug B, Engesaeter LB, Vollset SE, Havelin LI, Langeland N. Antibiotic prophylaxis in total hip arthroplasty. Review of 10,905 primary cemented total hip replacements reported to the Norwegian arthroplasty register, 1987 to 1995. J Bone Joint Surg Br. 1997 Jul;79(4):590-95.

43. Kweon C, McLaren AC, Leon C, McLemore R. Amphotericin B delivery from bone cement increases with porosity but strength decreases. Clin.Orthop. 2011 Nov;469(11):3002-3007.
44. Buranapanitkit B, Oungbho K, Ingviya N. The efficacy of hydroxyapatite composite impregnated with amphotericin B. Clinical Orthopaedics \& Related Research 2005.

45. Sealy PI, Nguyen C, Tucci M, Benghuzzi H, Cleary JD. Delivery of antifungal agents using bioactive and nonbioactive bone cements. Ann Pharmacother. 2009 Oct;43(10):1606-15.

46. Silverberg D, Kodali P, Dipersio J, Acus R, Askew M. In vitro analysis of antifungal impregnated polymethylmethacrylate bone cement. Clin.Orthop. 2002 Oct;403:228-31.

47. Wu MH, Hsu KY. Candidal arthritis in revision knee arthroplasty successfully treated with sequential parenteral-oral fluconazole and amphotericin B-loaded cement spacer. Knee Surg.Sports Traumatol.Arthrosc. 2011 Feb;19(2):273-76.

48. Dellinger RP, Levy MM, Rhodes A, Annane D, Gerlach $\mathrm{H}$, Opal SM, et al. Surviving Sepsis Campaign: international guidelines for management of severe sepsis and septic shock, 2012. Intensive Care Med. 2013 Feb;39(2):165-228.

This article is also available online on the SAOA website (www.saoa.org.za) and the SciELO website (www.scielo.org.za). Follow the directions on the Contents page of this journal to access it. 